\title{
SUPERFIELD EQUATIONS OF MOTION
}

\author{
(Presented by H. Keres)
}

\begin{abstract}
The general form of massive superfield equations of motion is proposed, using the formalism of superprojectors. It is shown that the known equations for scalar and bispinor superfield mav be presented in a given general form. The possible equations for a vector superfield and scalar superfield which describe superspin $3 / 2$ and lower superspins, are given.
\end{abstract}

\section{Introduction}

The concept of superfield introduced in [ $\left.{ }^{1}\right]$ has proved to be a very useful object dealing with the supersymmetry. At the same time the superfield is a complicated object - it contains many ordinary Bose and Fermi fields. Although in applications we mostly deal with the ordinary component fields, we need the mathematical apparatus in order to manage with more compact expressions formulated with the help of superfields. Due to the fact that superfields contain representations with different superspins, we need analogical equations of motion like those in the ordinary field case. The role of the superfield equation of motion is to describe a supermultiplet with given mass and superspin or supermultiplets with several masses and superspins.

Some of the superfield equations of motion were previously treated in papers $\left[{ }^{2-6}\right]$. It appears that many of the general principles used in the theory of ordinary wave equations are also apdlicable in the superfield case. V. I. Ogievetsky and E. Sokatchev $\left[{ }^{4,5}\right]$ generalized the root method into the superfield case, and proposed equations for bispinor and vector superfields. Although the root method is applicable in the derivation of many superfield equations of motion, it is not the most general one. There are equations, as, for example, the Salam-Strathdee equation for chiral bispinor superfield, which is not derivable via the root method. In this paper we propose the general method for deriving superfield equations of motion that is based on the analogy of the derivation of ordinary wave equations with the help of covariant superprojectors. The most relevant objects in our construction are superprojectors $E_{i j}^{Y}$ which separate superspin $Y$ from the superfield $\Phi_{j}(x, \theta)$, and transform it to the superfield $\Phi_{i}(x, \theta)$. Operators $E_{i i}^{Y}$ are ordinary superprojectors the general expressions of which are well known, the problem is to find superspin transition operators $E_{i j}^{Y}(i \neq j)$. Here we give superspintransition operators for some most widely used representations.

The paper is planned as follows. At the beginning we give the general formalism for the derivation of superfield equations of motion in the formalism of superprojectors $E_{i j}^{Y}$, and then the schema to analyse the mass and superspin spectrum for a given equation is presented. Further; 
we demonstrate that the known superfield equations for scalar and bispinor superfields have the proposed general structure. We analyse more thoroughly the possible superfield equations for a vector superfield $h^{\mu}(x, \theta)$ and scalar superfield $\Phi(x, \theta)$, and investigate the mass spectrum of given equations. More interesting equations from the obtained ones are those which describe superspin $3 / 2$, since superspin $3 / 2$ contains Poincaré spins 2 and $3 / 2$ needed in the supergravity theory.

In this paper we deal only with the massive case. Although physically the zero rest mass equations are more interesting, it should be pointed out that the zero rest mass case is more easily obtained from the massive equations. Massive equations in hand, it remains to find equations invariant with respect to the gauge transformations when we set $m=0$. Here we do not give the equations in terms of component fields either since this procedure is not very complicated. As it is demonstrated in the bispinor superfield case $\left[{ }^{5,7}\right]$, the structure of component field equations is quite interesting and many of the well-known equations are obtained.

\section{General superfield equation of motion}

Here we give the general form of superfield equations of motion. We consider some general superfield $\Phi(x, \theta)$. Firstly we deal with the representation where $\Phi(x, \theta)$ is expressed as a direct sum of superfields $\Phi_{i}(x, \theta)$ which transform in the Lorentz transformations according to the irreducible representation $i \equiv\left(k_{i}, l_{i}\right)$ of the Lorentz group. We assume that we have superprojectors $E_{i j}^{Y}$ which satisfy

$$
E_{i j}^{Y} E_{j k}^{Y^{\prime}}=\delta_{Y Y^{\prime}} E_{i k}^{Y}
$$

(over $j$ is not summed). Here $E_{i i}^{Y}$ are ordinary superprojectors that separate superspin $Y$ from the superfield $\Phi_{i}(x, \theta)$, and $E_{i j}^{Y}(i \neq j)$ are superspin-transition operators that separate superspin $Y$ from the superfield $\Phi_{j}(x, \theta)$, and transform it to the superfield $\Phi_{i}(x, \theta)$, i. e. the superfields $\Phi_{i}(x, \theta)$ and $E_{i j}^{Y} \Phi_{j}(x, \theta)$ transform according to the same representation $i$.

The general $n$-th order superfield equation of motion for $\Phi(x, \theta)$ is written in the following form

$$
(-\square)^{n / 2} \pi \Phi(x, \theta)=m^{n} \Phi(x, \theta),
$$

where

$$
\pi=\left|\begin{array}{cccc}
a_{11} E_{11} & a_{12} E_{12} & \ldots & a_{1 r} E_{1 r} \\
a_{21} E_{21} & a_{22} E_{22} & \ldots & a_{2 r} E_{2 r} \\
\cdot & \cdot & & \cdot \\
\cdot & \cdot & & \cdot \\
a_{r 1} E_{r 1} & a_{r 2} \dot{E}_{r 2} & \ldots & a_{r r} E_{r r}
\end{array}\right|, \quad \Phi=\left|\begin{array}{c}
\Phi_{1} \\
\Phi_{2} \\
\cdot \\
\dot{\Phi_{r}}
\end{array}\right|
$$

and $a_{i j}$ are arbitrary parameters. Operators $E_{i j}$ are the following

$$
E_{i j}=\sum_{Y} \alpha_{i j}(Y) E_{i j}^{Y} .
$$

We have no general expressions of $\alpha_{i j}(Y)$, and for that reason the following rule is useful: $\alpha_{i j}(Y)$ must be chosen so that for a $n$-th order equation maximal nonlocality of all operators $E_{i j}$ is not higher than $\square^{-n / 2}$. This condition restricts the choice of representations $i$ and $j$, and, similarly, as in the case of ordinary wave equations, we say that the representations $i$ and $j$ are linked if the operator $E_{i j}$ with needed non- 
locality exists. Otherwise the representations $i$ and $j$ are not linked, and in (2.3) one must set $a_{i j}=0$. Without any loss of generality, we also set $\alpha_{i j}(Y)$ corresponding to the maximal common superspin $Y$ in $(2.4)$ equal to one, i. e. $\alpha_{i j}\left(Y_{\max }\right)=1$.

The analysis of mass and superspin spectrum is performed as follows. We decompose

$$
\pi=\pi^{Y_{1}}+\pi^{Y_{2}}+\ldots+\pi^{Y_{k}},
$$

where $\pi^{Y}$ contains projectors $E_{i j}^{Y}$ of a given superspin $Y$. Masses corresponding to a superspin $Y$ are determined with the help of nonzero eigenvalues of reduced matrix $\pi_{Y}$ formed from the coefficients

$$
\pi_{Y}=\left|\begin{array}{cccc}
a_{11} \alpha_{11}(Y) & a_{12} \alpha_{12}(Y) & \ldots & a_{1 r} \alpha_{1 r}(Y) \\
a_{21} \alpha_{21}(Y) & a_{22} \alpha_{22}(Y) & \ldots & a_{2 r} \alpha_{2 r}(Y) \\
\cdot & \cdot & & \vdots \\
\cdot & \cdot & & \vdots \\
a_{r 1} \alpha_{r 1}(Y) & a_{r 2} \alpha_{r 2}(Y) & \ldots & a_{r r} \alpha_{r r}(Y)
\end{array}\right|
$$

as follows: $m_{k}=m\left(\lambda_{k}\right)^{-1 / n}$, where $\lambda_{k}$ are some nonzero eigenvalue of $\pi_{Y}$. Indeed, using (2.1) we obtain that $\pi_{Y}$ satisfies the same minimal polynomial as $\pi^{Y}$, and has therefore the same nonzero eigenvalues as $\pi^{Y}$. From (2.1) we also have $\pi^{Y} \pi^{Y^{\prime}}=\delta_{Y Y^{\prime}}\left(\pi^{Y}\right)^{2}$.

The operator $\pi$ contains, in general, operators $\pi^{Y}$ of superspins $Y$, which we do not want to describe. Similarly, as in the case of ordinary wave equations, one should require that these $\pi^{Y}$ are nilpotent, i.e. $\left(\pi^{Y}\right)^{a}=0, a \geqslant 2$.

While exploiting the general schema $(2.2)-(2.4)$, the main problem is how to find superspin-transition operators $E_{i j}^{Y}$. In the following we give operators $E_{i j}^{Y}$ or some mostly used superfields. The expressions of superprojectors $E_{i i}^{Y}$ are given in Appendix. In the scalar superfield case the projectors were given in $\left[^{2}\right]$, in the case of symmetrical tensor and tensor-bispinor superfields, the projectors were given in $\left[{ }^{8}\right]$, in $\left[{ }^{9}\right]$ the results of $\left.{ }^{8}\right]$ were generalised to an arbitrary superfield case.

In some cases the equation is presented in the form where $\Phi(x, \theta)$ is decomposed into a direct sum of superfields $\Phi_{\alpha}(x, \theta)$ which are not irreducible under the Lorentz transformations. Then it is useful to distribute the operators into two classes: projectors $E_{\alpha \beta}^{Y}$ and operators $\beta_{\alpha \beta}^{Y}$, which are used in equation. Operators $\beta_{\alpha \beta}^{Y}$ connect the linked representations, and $E_{\alpha \beta}^{Y}$ connect the representations that are not linked. In the terminology of the root method $\left[{ }^{5}\right]$, the operators $\beta_{\alpha \beta}^{Y}$ are roots of projectors $E_{\alpha \beta}^{Y}$. Operators $\beta_{\alpha \beta}^{Y}$ satisfy the relations

$$
\begin{gathered}
\beta_{\alpha \beta}^{Y} E_{\beta \gamma}^{Y^{\prime}}=E_{\alpha \beta}^{Y} \beta_{\beta \gamma}^{Y^{\prime}}=\delta_{Y Y^{\prime}} \beta_{\alpha \gamma}^{Y} \\
\beta_{\alpha \beta}^{Y} \beta_{\beta \gamma}^{Y^{\prime}}=\delta_{Y Y^{\prime}} E_{\alpha \gamma}^{Y} .
\end{gathered}
$$

The general $n$-th order equation is presented in the same form (2.2), but now

$$
\pi=\left|\begin{array}{ccc}
a_{11} \beta_{11} & \ldots & a_{1 \rho} \beta_{1 \rho} \\
\cdot & & \cdot \\
\cdot & & \cdot \\
a_{\rho 1} \beta_{\rho 1} & \ldots & a_{\rho \rho} \beta_{\rho \rho}
\end{array}\right|, \quad \Phi=\left|\begin{array}{c}
\Phi_{1} \\
\cdot \\
\dot{\Phi_{\rho}}
\end{array}\right|
$$

and 


$$
\beta_{\alpha \beta}=\sum_{Y} \alpha_{\alpha \beta}(Y) \beta_{\alpha \beta}^{Y} .
$$

It should be noted that the general structures (2.3) and (2.8) may be mixed with some matrix algebra, and then the given structure is somewhat masked. In the spinor superfield case, for example, the general structure has been mixed with the Dirac $\gamma$-algebra, and instead of (2.8) we have $\hat{\partial} \pi$ (see $\$ 4)$.

Concluding this paragraph, we note that the physical mass spectrum may be obtained from the first or second-order equations, since for $n>2$, among the roots $(\lambda)^{-1 / n}$, are also the complex ones. The general form of first-order equation is from $(2.2)$

$$
i \sqrt{\square} \pi \Phi(x, \theta)=m \Phi(x, \theta),
$$

where $\alpha_{i j}(Y)$ in $(2.4)$ or $(2.9)$ must be chosen so that the nonlocality of $E_{i j}$ or $\beta_{\alpha \beta}$ is $1 / \sqrt{\sqcup}$. The general form of a second-order equation is

$$
\square \pi \Phi(x, \theta)+m^{2} \Phi(x, \theta)=0 .
$$

In the following two paragraphs we demonstrate that the proposed general schema works in the case of known equations for chiral scalar superfield $\Phi_{ \pm}(x, \theta)$ and bispinor superfield $\psi_{\alpha}(x, \theta)$ given in $\left[{ }^{2,4-6}\right]$.

\section{Chiral scalar superfield}

We shall deal with the description of superspin 0 with the help of two chiral superfields: $\Phi_{1}(x, \theta)=\Phi_{+}(x, \theta)$ and $\Phi_{2}(x, \theta)=\Phi_{-}(x, \theta)$. It is possible to obtain two choices of projectors $E_{i j}^{u}$ (see Appendix for notations and projectors):

1) $E_{11}^{0}=E_{22}^{0} \equiv E_{0+}^{0}+E_{0-}^{0}=-\frac{d^{2}}{4 \square}$,

$$
E_{12}^{0}=E_{21}^{0}=-\frac{i}{2 \sqrt{\square}}\left(d \cos \xi+d^{5} \sin \xi\right),
$$

where $\xi$ is an arbitrary real parameter and $E_{0 \pm}^{0}$ are given by (A. 3). Using (A. 1), it is possible to verify that $E_{i j}^{0}$ satisfy (2.1).

2) $E_{11}^{0} \equiv E_{0+}^{0}=-\frac{1}{8 \square}\left(d^{2}+2 i \partial_{\rho} d^{\rho}\right)$,

$$
\begin{aligned}
& E_{22}^{0} \equiv E_{0-}^{0}=-\frac{1}{8 \square}\left(d^{2}-2 i \partial_{\rho} d^{\rho}\right), \\
& E_{12}^{0}=-\frac{i}{4 \sqrt{\square}}\left(d-i d^{5}\right), \\
& E_{21}^{0}=-\frac{i}{4 \sqrt{\square}}\left(d+i d^{5}\right) .
\end{aligned}
$$

Operators $d \cos \xi+d^{5} \sin \xi$ and $\Gamma_{ \pm}=\frac{1}{4}\left(d \pm i d^{5}\right)$ used in (3.1), and were derived from the considerations of root method in $\left.{ }^{6}\right]$.

From (3.1) and (3.2) we see that $E_{12}^{0}$ and $E_{21}^{0}$ allow to obtain a first- 
order equation, since these operators have the needed nonlocality $1 / \sqrt{\square}$. Therefore, in the first-order equation the representations $\Phi_{+}$and $\Phi_{-}$are linked, whereas $\Phi_{+}$and $\Phi_{+}, \Phi_{-}$and $\Phi_{-}$, are not linked. where

The general first-order equation for $\Phi_{ \pm}$is written as (2.107),

$$
\pi \equiv \pi^{0}=\left|\begin{array}{cc}
0 & a_{12} E_{12}^{0} \\
a_{21} E_{21}^{0} & 0
\end{array}\right|, \quad \Phi=\left|\begin{array}{c}
\Phi_{+} \\
\Phi_{-}
\end{array}\right| .
$$

Without no loss of generality we may set $a_{12}=a_{21}=1$. Indeed, the mass is determined with the help of nonzero eigenvalues of the reduced matrix $\pi_{0}$

$$
\pi_{0}=\left|\begin{array}{cc}
0 & a_{12} \\
a_{21} & 0
\end{array}\right| .
$$

From (3.4) we have $\lambda^{2}=a_{12} a_{21}$. If we choose the mass of superspin 0 to be equal to $m$, we must set $\lambda= \pm 1$ and then, from the considerations of symmetry, we choose $a_{12}=a_{21}=1$.

Using the first choice of operators $E_{i j}^{0}$, the equation (2.10) takes, in the case of (3.3), the following form $\left[{ }^{6}\right]$

$$
\begin{aligned}
& \frac{1}{2}\left(d \cos \xi+d^{5} \sin \xi\right) \Phi_{-}=m \Phi_{+}, \\
& \frac{1}{2}\left(d \cos \xi+d^{5} \sin \xi\right) \Phi_{+}=m \Phi_{-} .
\end{aligned}
$$

Setting $\xi=0$, we obtain the equation given in $\left[{ }^{2}\right]$.

The second choice of operators $E_{i j}^{0}$ gives the equation

$$
\begin{aligned}
& \frac{1}{4}\left(d-i d^{5}\right) \Phi_{-}=m \Phi_{+}, \\
& \frac{1}{4}\left(d+i d^{5}\right) \Phi_{+}=m \Phi_{-} .
\end{aligned}
$$

Although the equations (3.5) and (3.6) are different, it is possible to verify that the equations for component fields are the same $\left.{ }^{6}\right]$.

\section{Bispinor superfield}

Bispinor superfield equations of motion for superspins 1,0 and $1 / 2$ given in $\left[{ }^{2,5,6}\right]$; show the general structure of $\S 2$, whereas in the superspin 1 and 0 cases the general structure has been mixed with the Dirac algebra.

4.1. Superspin 1. The superspin 1 equation given in $\left[{ }^{5}\right]$ is the following (without the loss of generality we set $\xi=\eta=0$ ) -

$$
\frac{1}{8}\left(6 i \hat{\partial}-i \gamma_{v} \gamma^{5} d^{v}+d+3 \gamma^{5} d^{5}\right) \Psi=m \Psi .
$$

The superspin content of equation (4.1) is obtained if we apply projectors (A. 5) $E^{1}, E_{+}^{1 / 2}+E_{-}^{1 / 2}, E^{0}$. If we write $(4.1)$ as

$$
i \sqrt{\square}\left(\pi^{1}+\pi^{1 / 2}+\pi^{0}\right) \Psi=m \Psi,
$$

we get 


$$
\begin{aligned}
& \pi^{1}=\frac{\hat{\partial}}{\sqrt{\square}} E^{1}=\frac{1}{\sqrt{\square}}\left[\frac{1}{8}\left(6 \hat{\partial}-\gamma_{\nu} \gamma^{5} d^{v}\right)+\frac{\hat{\partial}}{16 \square}\left(3 d^{2}+2 \gamma^{5} \partial_{\rho} d^{\rho}\right)\right], \\
& \pi^{1 / 2}=-\frac{1}{\sqrt{\square}}\left[\frac{i}{8}\left(d+3 \gamma^{5} d^{5}\right)+\frac{\hat{\partial}}{16 \square}\left(3 d^{2}+2 \gamma^{5} \partial_{\rho} d^{\rho}\right)\right], \\
& \pi^{0}=0 .
\end{aligned}
$$

As we can see, the $Y=1$ equation contains operators of superspins 1 and $1 / 2$. Equation (4.1) does not describe superspin $1 / 2$ because operator $\pi^{1 / 2}$ is nilpotent: $\left(\pi^{1 / 2}\right)^{2}=0 . \pi^{1}$ satisfies $\left(\pi^{1}\right)^{2}=E^{1}$. Our equation is represented in the form $(2.8)$, since we may write $\pi^{1} \equiv \beta^{1}=\frac{\hat{\partial}}{\sqrt{\square}} E^{1}$. Operator $\pi^{1 / 2}$ is needed to eliminate the nonlocal terms $\square^{-3 / 2}$ in $\pi^{1}$.

From (4.3) it is not obvious that $\pi^{1 / 2}$ may be represented with the help of projectors $E_{i j}^{Y}$, but if we write $\psi$ as a direct sum of two representations $\psi_{1}$ and $\psi_{2}$ which transform according to the spinor representations $(1 / 2,0)$ and $(0,1 / 2)$, and use the following representation of $\gamma$-matrices

$$
\gamma^{\mu}=\left(\begin{array}{cc}
0 & \sigma^{\mu} \\
\tilde{\sigma}^{\mu} & 0
\end{array}\right)
$$

we obtain $\pi^{1 / 2} \equiv \beta^{1 / 2}$ in the following form -

$$
\beta^{1 / 2}=\frac{\hat{\partial}}{2 \sqrt{\square}}\left|\begin{array}{cc}
2 E_{11}^{0}+E_{22}^{0} & \frac{\partial_{1}}{\sqrt{\square}}\left(2 E_{12}^{0}-E_{21}^{0}\right) \\
\frac{\partial_{2}}{\sqrt{\square}}\left(2 E_{21}^{0}-E_{12}^{0}\right) & E_{11}^{0}+2 E_{22}^{0}
\end{array}\right| .
$$

Here $\partial_{1}=\partial_{\mu} \sigma^{\mu}, \partial_{2}=\partial_{\mu} \tilde{\sigma}^{\mu}$ and $E_{i j}^{0}$ are operators (3.2).

4.2 Superspin 0 . The superspin 0 equation of motion given in $\left[{ }^{6}\right]$ is written in the following form (we also set $\xi=\eta=0$ ) -

$$
\frac{1}{8}\left(2 i \hat{\partial}+i \gamma_{v} \gamma^{5} d^{v}+d-\gamma^{5} d^{5}\right) \Psi=m \Psi .
$$

If we write (4.5) as (4.2), we get

$$
\begin{aligned}
& \pi^{0}=\frac{\hat{\partial}}{\sqrt{\square}} E^{0}=\frac{1}{\sqrt{\square}}\left[\frac{1}{8}\left(2 \hat{\partial}+\gamma_{v} \gamma^{5} d^{v}\right)+\frac{\hat{\partial}}{16 \square}\left(d^{2}-2 \gamma^{5} \partial_{\rho} \partial^{\rho}\right)\right], \\
& \pi^{1 / 2}=-\frac{1}{\sqrt{\square}}\left[\frac{i}{8}\left(d-\gamma^{5} d^{5}\right)+\frac{\hat{\partial}}{16 \square}\left(d^{2}-2 \gamma^{5} \partial_{\rho} d^{\rho}\right)\right], \\
& \pi^{1}=0 .
\end{aligned}
$$

Equation (4.5) describes only superspin 0 , since $\pi^{1 / 2}$ is nilpotent: $\left(\pi^{1 / 2}\right)^{2}=0$. If we take $\pi^{0} \equiv \beta^{0}$, we have $\left(\beta^{0}\right)^{2}=E^{0}$. Similarly, as in the superspin 1 case, operator $\pi^{1 / 2}$ eliminates the nonlocal terms $\square^{-3 / 2}$ in $\pi^{0} \cdot \pi^{1 / 2} \equiv \beta^{1 / 2}$ is expressed with the help of operators (3.2) in the following form - 


$$
\beta^{1 / 2}=\frac{\hat{\partial}}{2 \sqrt{\square}}\left|\begin{array}{cc}
E_{22}^{0} & \frac{\partial_{1}}{\sqrt{\square}} E_{21}^{0} \\
\frac{\partial_{2}}{\sqrt{\square}} E_{12}^{0} & E_{11}^{0}
\end{array}\right| .
$$

4.3 Superspin $1 / 2$. Superspin $1 / 2$ is described by the Salam-Strathdee equation for chiral bispinor superfields $\psi+$ and $\psi-\left[{ }^{2}\right]-$

$$
\frac{1}{4}\left|\begin{array}{cc}
2 i \hat{\partial} & d \\
d & 2 i \hat{\partial}
\end{array}\right|\left|\begin{array}{c}
\psi_{+} \\
\psi_{-}
\end{array}\right|=m\left|\begin{array}{c}
\psi_{+} \\
\psi_{-}
\end{array}\right| .
$$

The Salam-Strathdee equation has a structure (2.8) where $a_{11}=a_{12}=$ $=a_{21}=a_{22}=1 / 2$

$$
\frac{i \sqrt{\square}}{2}\left|\begin{array}{cc}
\beta_{11}^{1 / 2} & \beta_{12}^{1 / 2} \\
\beta_{21}^{1 / 2} & \beta_{22}^{1 / 2}
\end{array}\right|\left|\begin{array}{l}
\psi_{+} \\
\psi_{-}
\end{array}\right|=m\left|\begin{array}{l}
\psi_{+} \\
\psi_{-}
\end{array}\right| .
$$

Indeed, if we define $\beta_{i j}^{1 / 2}$ as

$$
\beta_{11}^{1 / 2}=\beta_{22}^{1 / 2}=\frac{\hat{\partial}}{\sqrt{\square}}, \quad \beta_{12}^{1 / 2}=\beta_{21}^{1 / 2}=-\frac{i d}{2 \sqrt{\square}},
$$

the operators $\beta_{i j}^{1 / 2}$ satisfy (2.7), while in the case of chiral fields one must use the identity $d^{2}=-4 \square$.

The equation (4.8) may be also represented in the form $(2.3)$ if we decompose $\psi_{+}=\psi_{+}^{1} \oplus \psi_{+}^{2}, \psi_{-}=\psi_{-}^{3} \oplus \psi_{-}^{4}$, where $\psi_{+}^{1}$ and $\psi_{-}^{3}$ transform according to the representation $(1 / 2,0)$ and $\psi_{+}^{2}, \psi_{-}^{4}$ according to $(0,1 / 2)$. From (4.8) we obtain

$$
\frac{i \sqrt{\square}}{2}\left|\begin{array}{cccc}
0 & E_{12}^{1 / 2} & E_{13}^{1 / 2} & 0 \\
E_{21}^{1 / 2} & 0 & 0 & E_{24}^{1 / 2} \\
E_{31}^{1 / 2} & 0 & 0 & E_{34}^{1 / 2} \\
0 & E_{42}^{1 / 2} & E_{43}^{1 / 2} & 0
\end{array}\right|\left|\begin{array}{c}
\psi_{+}^{1} \\
\psi_{+}^{2} \\
\psi_{-}^{3} \\
\psi_{-}^{4}
\end{array}\right|=m\left|\begin{array}{c}
\psi_{+}^{1} \\
\psi_{+}^{2} \\
\psi_{-}^{3} \\
\psi_{-}^{4}
\end{array}\right|,
$$

where

$$
\begin{gathered}
E_{12}^{1 / 2}=E_{34}^{1 / 2}=\frac{\partial_{1}}{\sqrt{\square}} ; \quad E_{21}^{1 / 2}=E_{43}^{11 / 2}=\frac{\partial_{2}}{\sqrt{\square}}, \\
E_{13}^{1 / 2}=E_{31}^{1 / 2}=E_{24}^{1 / 2}=E_{42}^{1 / 2}=-\frac{i d}{2 \sqrt{\square}} .
\end{gathered}
$$

It is interesting to note that each of the components is linked with the component of the same chirality via the Dirac algebra, and with the component of opposite chirality via the operator $d$. As we have already mentioned, the Salam-Strathdee equations is not derivable by the root method, since $\pi^{2} \neq E^{1 / 2}$, but we have $\pi^{3}=\pi$, which means that the eigenvalues of $\pi$ are equal to \pm 1 and 0 .

In conclusion of this paragraph we would like to give one possible realisation of operators $\beta^{Y}$. In the case of $Y=1$ and $Y=0$ equations we have used. 


$$
\beta^{1}=\frac{\hat{\partial}}{\sqrt{\square}} E^{1}, \quad \beta^{0}=\frac{\hat{\partial}}{\sqrt{\square}} E^{0} .
$$

It is also possible to give operators

$$
\beta_{+}^{1 / 2}=\frac{\hat{\partial}}{\sqrt{\square}} E_{+}^{1 / 2}, \quad \beta_{-}^{1 / 2}=\frac{\hat{\partial}}{\sqrt{\square}} E_{-}^{1 / 2} .
$$

All the operators $\beta^{Y}$ have a common quality $\left(\beta^{Y}\right)^{2}=E^{Y}$, i. e. they are roots of corresponding projection operators.

\section{Vector superfield and scalar superfield}

In the following, we deal with the equations that describe superspin $3 / 2$. Superspin $3 / 2$ contains Poincaré spins $2,3 / 2$, and for that reason the equations obtained may be useful, in the zero rest mass case, in theories of linearised supergravities. The lowest superfield containing superspin $3 / 2$, is the vector superfield $h^{\mu}(x, \theta)$. It appears that in addition to the vector superfield some other superfield is needed, and the most natural choice is the scalar superfield $\Phi(x, \theta)$.

We shall deal with the equations for superfields $\Phi_{1}(x, \theta)=h^{\mu}(x, \theta)$ and $\Phi_{2}(x, \theta)=\Phi(x, \theta)$. Firstly, we shall deal with the vector superfield $h^{\mu}(x, \theta)$. Superspin $3 / 2$ is extracted with the help of projector $E_{1}^{y / 2}$ (A.9), which we denote as $E_{11}^{3 / 2}$

$$
\left(E_{11}^{3 / 2}\right)^{x_{\lambda}}=\frac{2}{3}\left(1+\frac{d^{2}}{4 \square}\right)\left(\eta^{\varkappa_{\lambda}}-\frac{\partial^{\varkappa} \partial_{\lambda}}{\square}\right)-\frac{1}{6 \square} \varepsilon_{\lambda, \rho \sigma}^{\varkappa} \partial^{\rho} d^{\sigma} .
$$

Operator $E_{11}^{1 / 2}$ contains nonlocal terms $\square^{-1}$ and $\square^{-2}$. Using other projectors (A.9) and (A. 10), it is possible to eliminate $\square^{-2}$, and to obtain a. second-order equation (2.11). Similarly to $\left[{ }^{4,10}\right]$, we use the operators corresponding to the lowest superspin 0. From (A.10) we can see that one must take operator $E_{0+}^{0}+E_{0-}^{0}$, because in (5.1) there are no terms with $\partial_{\rho} d^{\rho}$. We denote $E_{0+}^{0}+E_{0-}^{0}=E_{11}^{0}$ and from (A. 10)

$$
\left(E_{11}^{0}\right)^{x_{\lambda}}=-\frac{d^{2}}{4 \square} \frac{\partial^{x} \partial_{\lambda}}{\square} .
$$

It is easy to verify that the operator $E_{11}=E_{11}^{3 / 2}-\frac{2}{3} E_{11}^{0}$ has the needed nonlocality $\square^{-1}$. The same operator was derived by the root method in $\left[{ }^{4,10}\right]$.

If we use only a vector superfield $h^{\mu}(x, \theta)$, we obtain the following equation $[4,10]-$

$$
\square\left(E_{11}^{3 / 2}-\frac{2}{3} E_{11}^{0}\right){ }^{\times} h^{\lambda}+m^{2} h^{*}=0 .
$$

Equation (5.3) describes superspin $3 / 2$ with mass $m$ and superspin 0 with nonphysical mass $i m \sqrt{7^{3 / 2}}$.

In order to eliminate superspin 0 , or to describe it by physical mass spectrum, we must take one more superfield which contains superspin 0 . The lowest superfield containing superspin 0 is the scalar superfield, and for that reason the second superfield we used is the scalar superfield 
$\Phi(x, \theta)$. Similarly to $(5.2)$, we take operator $E_{+}^{0}+E_{-}^{0}$ (A. 3), which we denote as $E_{22}^{0}$ -

$$
E_{22}^{0}=-\frac{d^{2}}{4 \square}
$$

5.1. Superspin $3 / 2$ and 0 equations Superfields $h^{\mu}(x, \theta)$ and $\Phi(x, \theta)$ are linked with the help of operators $E_{12}^{0}$ and $E_{21}^{0}$ which satisfy (2.1). It is easy to verify that the operators $E_{12}^{0}$ and $E_{21}^{0}$ are the following -

$$
\left(E_{12}^{0}\right)^{x}=\frac{d \partial^{\varkappa}}{2 \square}, \quad\left(E_{21}^{0}\right)_{\lambda}=-\frac{d \partial_{\lambda}}{2 \square} .
$$

The most general equation for superspins $3 / 2$ and 0 is, from $(2.3)$, the following -

$$
\square\left|\begin{array}{cc}
\left(E_{11}^{3 / 2}-\frac{2}{3} E_{11}^{0}\right)_{\lambda} \varkappa_{\lambda} \cdot a\left(E_{12}^{0}\right)^{\varkappa} \\
b\left(E_{21}^{0}\right)_{\lambda} & c E_{22}^{0}
\end{array}\right|\left|\begin{array}{c}
h^{\lambda} \\
\Phi
\end{array}\right|+m^{2}\left|\begin{array}{c}
h^{\kappa} \\
\Phi
\end{array}\right|=0,
$$

where we have denoted $a_{11}=1, a_{12}=a, a_{21}=b$ and $a_{22}=c$.

Using the expressions of operators $E_{i j}^{Y}$; (5.6) may be written as

$$
\begin{aligned}
& \frac{2}{3}\left[\left(\square+\frac{d^{2}}{4}\right) h^{\varkappa}-\partial^{\varkappa} \partial_{\lambda} h^{\lambda}\right]-\frac{1}{6} \varepsilon_{\lambda \rho \sigma}^{\varkappa} \partial^{\rho} d^{\sigma} h^{\lambda}+\frac{a}{2} d \partial^{\varkappa} \Phi+m^{2} h^{\varkappa}=0, \\
& -\frac{b}{2} d \partial_{\lambda} h^{\lambda}-\frac{c}{4} d^{2} \Phi+m^{2} \Phi=0 .
\end{aligned}
$$

Equation (5.6) describes superspin $3 / 2$ with mass $m$ and, depending on the choice of parameters $a, b$ and $c$, superspin 0 states with different masses. It is possible to choose the coefficients so that (5.6) describes single superspin $3 / 2$.

The analysis of mass spectrum is the following: at first we decompose $\pi=\pi^{3 / 2}+\pi^{0}$

$$
\pi^{3 / 2}=\left|\begin{array}{cc}
E_{11}^{3 / 2} & 0 \\
0 & 0
\end{array}\right|, \quad \pi^{0}=\left|\begin{array}{cc}
-\frac{2}{3} E_{11}^{0} & a E_{12}^{0} \\
b E_{21}^{0} & c E_{22}^{0}
\end{array}\right| .
$$

Now the analysis reduces to the investigation of nonzero eigenvalues of reduced matrices $\pi_{3 / 2}$ and $\pi_{0}-$

$$
\pi_{3 / 2}=\left|\begin{array}{ll}
1 & 0 \\
0 & 0
\end{array}\right|, \quad \pi_{0}=\left|\begin{array}{cc}
-2 / 3 & a \\
b & c
\end{array}\right| .
$$

The only nonzero eigenvalue of $\pi_{3 / 2}$ is equal to one, and therefore the mass of superspin $3 / 2$ is equal to $m$. The masses of superspin 0 states $m / \sqrt{\lambda_{1}}$ and $m / \sqrt{\lambda_{2}}$ are obtained from the eigenvalues of $\pi_{0}$ that are calculated from

$$
6 \lambda_{1,2}=3 c-2 \pm \sqrt{(3 c+2)^{2}+36 a b} .
$$

The masses of superspin 0 states, therefore, depend on two free parameters $a b$ and $c$. In order to have physical mass spectrum, $\lambda_{1}$ and $\lambda_{2}$ must be real and nonnegative. These conditions are guaranteed if $a b$ and $c$ satisfy

$$
c \geqslant 2 / 3, \quad 3 a b+2 c \leqslant 0, \quad(3 c+2)^{2}+36 a b \geqslant 0 .
$$


The region of parameters determined by (5.11) we call a physical region. It is useful to represent the possible values of parameters on the

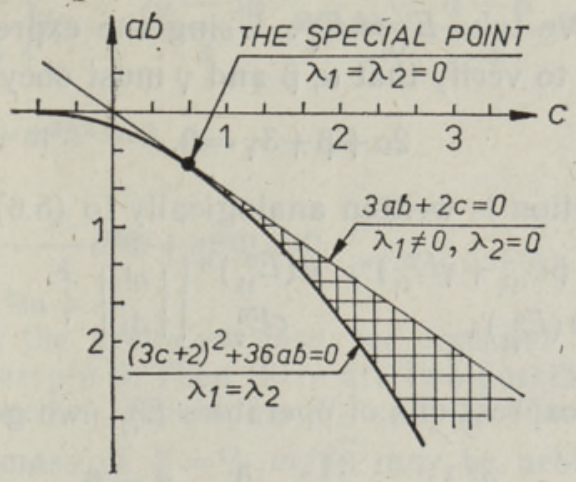

Superfield equations of motion

$a b-c$ diagram (Figure), where the shaded region corresponds to the physical region. The physical region is determined by the line

$$
3 a b+2 c=0,
$$

which gives the eigenvalues

$$
\lambda_{1}=c-2 / 3, \quad \lambda_{2}=0,
$$

and by the parabola

$$
(3 c+2)^{2}+36 a b=0,
$$

which gives coincident eigenvalues

$$
\lambda_{1}=\lambda_{2}=(3 c-2) / 6 .
$$

The points on the line (5.12), in addition to superspin $3 / 2$ describe two superspins 0 with mass $m / \sqrt{\lambda_{1}}$, two superspins 0 are there due to $\lambda_{2}=0$ being eliminated. $\pi_{0}$ satisfies the minimal equation $\pi_{0}\left(\pi_{0}-\lambda_{1}\right)=0$.

The points on the parabola (5.14) we regard analogically; as in the case of ordinary wave equations unphysical, since $\pi_{0}$ satisfies the minimal equation $\left(\pi_{0}-\lambda_{1}\right)^{2}=0$, and, for that reason, has only one nonzero eigenvector. Only one of the points on the parabola is physically interesting. It corresponds to the eigenvalues $\lambda_{1}=\lambda_{2}=0$, and describes single superspin $3 / 2$. From (5.14) and (5.15) single superspin $3 / 2$ is described by the parameters

$$
a b=-4 / 9, \quad c=2 / 3 .
$$

The other points in the physical region give $\lambda_{1} \neq \lambda_{2}$, and describe superspin 0 states with masses $m / \sqrt{\lambda_{1}}$ and $m / \sqrt{\lambda_{2}}$. It is interesting to note that these masses may have arbitrary values.

The most interesting equation from the equations we dealt with in this section is the equation for single superspin $3 / 2$ corresponding to a special point (5.16). The other equations describe, in addition to superspin $3 / 2$, at least two superspins 0 with the same mass, since the operators $E_{11}^{0}$ and $E_{22}^{0}$ separate two superspins 0 from both representations.

5.2. Superspins $3 / 2$ and $1 / 2$. The schema used in 5.1 is applicable for the description of superspins $3 / 2$ and $1 / 2$, or $3 / 2,1 / 2$ and 0 . We represent $E_{11}$ in the following form

$$
E_{11}=\alpha E_{11}^{3 / 2}+\beta E_{11}^{1 / 2}+\gamma E_{11}^{0},
$$


and choose $\alpha, \beta$ and $\gamma$ so that $E_{11}$ has nonlocality $\square^{-1}$. Since we have two superspin projectors - $E_{1}^{1 / 2}(A .9)$ and $E_{0}^{1 / 2}(A .10)$, we get two different equations. We shall consider them separately.

5.2a Operator $\boldsymbol{E}_{1}^{1 / 2}$. We take $E_{11}^{1 / 2} \equiv E_{1}^{1 / 2}$. Using the expressions (5.1),

and (A.9), it is easy to verify that $\alpha, \beta$ and $\gamma$ must obey

$$
2 \alpha+\beta+3 \gamma=0 \text {. }
$$

The general equation is written analogically to (5.6) as

$$
\square\left|\begin{array}{cc}
\left(\alpha E_{11}^{1 / 2}+\beta E_{11}^{1 / 2}+\gamma E_{11}^{0}\right)^{\kappa_{\lambda}} & a\left(E_{12}^{0}\right)^{\kappa} \\
b\left(E_{21}^{0}\right) \lambda & c E_{22}^{0}
\end{array}\right|\left|\begin{array}{c}
h^{\lambda} \\
\Phi
\end{array}\right|+m^{2}\left|\begin{array}{c}
h^{\varkappa} \\
\Phi
\end{array}\right|=0 .
$$

Using the given expressions of operators $E_{i j}^{Y}$, we get

$$
\begin{aligned}
& \frac{2 \alpha+\beta}{3}\left[\left(\square+\frac{d^{2}}{4}\right) h^{\varkappa}-\partial^{\varkappa} \partial_{\lambda} h^{\lambda}\right]-\frac{\alpha-\beta}{6} \varepsilon_{\lambda \rho \sigma}^{\lambda_{\alpha}} \partial \rho d^{\sigma} h^{\lambda}+ \\
& +\frac{a}{2} d \partial^{\varkappa} \Phi+m^{2} h^{\varkappa}=0, \\
& -\frac{b}{2} d \partial_{\lambda} h^{\lambda}-\frac{c}{4} d^{2} \Phi+m^{2} \Phi=0 .
\end{aligned}
$$

Decomposing $\pi=\pi^{3 / 2}+\pi^{1 / 2}+\pi^{0}$, we obtain

$$
\pi^{3 / 2}=\left|\begin{array}{cc}
\alpha E_{11}^{3 / 2} & 0 \\
0 & 0
\end{array}\right|, \quad \pi^{1 / 2}=\left|\begin{array}{cc}
\beta E_{11}^{1 / 2} & 0 \\
0 & 0
\end{array}\right|, \quad \pi^{0}=\left|\begin{array}{cc}
\gamma E_{11}^{0} & a E_{12}^{0} \\
b E_{21}^{0} & c E_{22}^{0}
\end{array}\right| .
$$

As we see, we can take $\alpha$ and $\beta$ as free parameters that determine $Y=3 / 2$ mass $m / \sqrt{\alpha}$, and $Y=1 / 2$ mass $m / \sqrt{\beta}$. The choice of $\alpha$ and $\beta$ determines, from (5.18), $\gamma$, and now, by the proper choice of $a b$ and $c$, it is possible to determine the masses corresponding to superspin 0 . Here we consider the case when $Y=0$ is eliminated. Then $\pi^{0}$ is nilpotent and equation (5.19) describes superspins $3 / 2$ and $1 / 2$, or $1 / 2$.

1. Equation describes superspins $3 / 2$ and $1 / 2$. Without any loss of generality, we may take $\alpha=1$ which gives the $Y=3 / 2$ mass $m$. The mass of $Y=1 / 2$ state $m / \sqrt{\beta}$ may be arbitrary. In order to have physical masses $\beta>0, \beta=0$ leads to the case treated in (5.1). From $(5.18)$

$$
\gamma=-(2+\beta) / 3
$$

and $\pi^{0}$ is nilpotent if

$$
a b=-\gamma^{2}, \quad c=-\gamma .
$$

2. Equation describes only superspin $1 / 2$. In that case we must set $\alpha=0$ and, without any loss of generality, we may choose $\beta=1$ which gives $Y=1 / 2$ mass $m$. Now $\gamma=-1 / 3$ and

$$
a b=-1 / 9, \quad c=1 / 3 .
$$

The equations in both cases are obtained from (5.20) if we choose the corresponding $\alpha, \beta, \gamma, a b$ and $c$.

5.2b Operator $\boldsymbol{E}_{0}^{1 / 2}$. Choosing $E_{11}^{1 / 2}=E_{0}^{1 / 2}$ we obtain from (5.1), (5.2) and (A.9)

$$
2 \alpha-3 \beta+3 \gamma=0 \text {. }
$$


The general equation remains the same $-(5.19)$, and gives in the case of our $E_{i j}^{Y}$

$$
\begin{aligned}
& \frac{2 \alpha}{3}\left(\square+\frac{d^{2}}{4}\right) h^{\kappa}-\frac{2 \alpha-3 \beta}{3} \partial^{\kappa} \partial_{\lambda} h^{\lambda}-\frac{\alpha-\beta}{6} \varepsilon_{\lambda \rho \sigma}^{\kappa} \partial^{\rho} d^{\sigma} h^{\lambda}+ \\
& +\frac{a}{2} d \partial^{\varkappa} \Phi+m^{2} h^{\kappa}=0 \\
& -\frac{b}{2} d \partial_{\lambda} h^{\lambda}-\frac{c}{4} d^{2} \Phi+m^{2} \Phi=0 .
\end{aligned}
$$

Similarly, as in the previous case, we consider the equations that do not describe superspin 0 . Then there are two possibilities:

1. Equation describes superspins $3 / 2$ and $1 / 2$. Choosing $\alpha=1 \quad(Y=3 / 2$ has mass $m$ ), the mass of $Y=1 / 2 m / \sqrt{\beta}$ may be arbitrary. From (5.25),

$$
\gamma=(3 \beta-2) / 3 \text {, }
$$

and $\pi^{0}$ is nilpotent when $a b$ and $c$ satisfy (5.23).

In the case of $\beta=2 / 3$ we get $\gamma=0$, and it is possible to take $a=b=$ $=c=0$, i. e. it is possible to use vector superfield $h^{\mu}(x, \theta)$ only.

2. Equation describes superspin $1 / 2$. In that case $\alpha=0, \beta=1$ and $\gamma=1 . a b$ and $c$ must satisfy

$$
a b=c=-1 .
$$

5.3 Superspins $3 / 2$ and 1 . The schema proposed in 5.1 is suitable also in the cases of superspins $3 / 2$ and 1 or $3 / 2,1$ and 0 . Operator $E_{11}^{1}$ must be chosen equal to $E_{1+}^{1}+E_{1-}^{1}$ since $E_{11}^{3 / 2}$ and $E_{11}^{0}$ do not contain $\partial_{\rho} d^{\rho}$. From (A.9),

$$
\left(E_{11}^{1}\right)^{x_{\lambda}}=-\frac{d^{2}}{4 \square}\left(\eta^{\varkappa_{\lambda}}-\frac{\partial^{\varkappa} \partial_{\lambda}}{\square}\right) .
$$

Operator $E_{11}$ can be presented as

$$
E_{11}=\alpha E_{11}^{3 / 2}+\beta E_{11}^{1}+\gamma E_{11}^{0},
$$

where $\alpha, \beta$ and $\gamma$ satisfy

$$
2 \alpha-3 \beta+3 \gamma=0 .
$$

The general equation is

$$
\square\left|\begin{array}{cc}
\left(\alpha E_{11}^{3 / 2}+\beta E_{11}^{1}+\gamma E_{11}^{0}\right)^{\varkappa} \lambda & a\left(E_{12}^{0}\right)^{\varkappa} \\
b\left(E_{21}^{0}\right)_{\lambda} & c E_{22}^{0}
\end{array}\right|\left|\begin{array}{c}
h^{\lambda} \\
\Phi
\end{array}\right|+m^{2}\left|\begin{array}{c}
h^{\varkappa} \\
\Phi
\end{array}\right|=0,
$$

or in greater detail

$$
\begin{aligned}
& \frac{2 \alpha}{3} \square h^{\alpha}+\frac{2 \alpha-3 \beta}{3}, \frac{d^{2}}{4} h^{\varkappa}-\frac{2 \alpha}{3} \partial^{\varkappa} \partial_{\lambda} h^{\lambda}- \\
& -\frac{\alpha}{6} \varepsilon_{\lambda \rho \sigma}^{\varkappa} \partial^{\rho} d^{\sigma} h^{\lambda}+\frac{a}{2} d \partial^{\varkappa} \Phi+m^{2} h^{\varkappa}=0, \\
& -\frac{b}{2} d \partial_{\lambda} h^{\lambda}-\frac{c}{4} d^{2} \Phi+m^{2} \Phi=0 .
\end{aligned}
$$

We shall treat only two cases: 
1. Superspins $3 / 2$ and 1 . We shall choose $\alpha=1$, which gives $Y=3 / 2$ mass $m$, superspin 1 has mass $m / \sqrt{\beta}$. From (5.31) we have $\gamma=$ $=(3 \beta-2) / 3$, and $\pi^{0}$ is nilpotent if $a b=-\gamma^{2}, c=-\gamma$.

2. Superspin 1. In order to describe superspin 1 one must choose $\alpha=0$. If we take $Y=1$ mass to be equal to $m$, we get $\beta=1$ and, therefore, $\gamma=1, a b=c=-1$.

In equations treated in this section all superspin 1 states are double, because operator $E_{11}^{1}$ extracts two chiral irreducible representations from the vector superfield.

5.4 Superspin 1 equations. In the previous sections of this paragraph we mostly dealt with the equations that described superspin $3 / 2$. By using the vector and scalar superfields, it is possible to give equations to describe lower superspins. Some of these equations were mentioned in 5.2 and 5.3, here we consider the superspin 1 case. In 5.3 there were two superspin 1 states, but if we do not describe superspin $3 / 2$, it is possible to choose operators $E_{1+}^{1}$ or $E_{1-1}^{1}$ separately as operators $E_{11}^{1}$.

Let the operator $E_{11}^{1}$ be equal to $E_{1+}^{1} ;($ A.9)

$$
\left(E_{11}^{1}\right)^{x_{\lambda}}=-\frac{1}{8 \square}\left(d^{2}+2 i \partial_{\rho} d^{\rho}\right)\left(\eta^{\varkappa_{\lambda}}-\frac{\partial^{x} \partial_{\lambda}}{\square}\right) .
$$

It is evident that one must use operator $E_{0+}^{0}$ in order to eliminate $\square^{-2}$ nonlocalities. Therefore

$$
\left(E_{11}^{0}\right)^{\kappa_{\lambda}}=-\frac{1}{8 \square}\left(d^{2}+2 i \partial_{\rho} d \rho\right) \frac{\partial^{\kappa} \partial_{\lambda}}{\square},
$$

and $E_{11}$ is equal to

$$
E_{11}=E_{11}^{1}+E_{11}^{0} .
$$

In the case of projectors $E_{i j}^{0}$, it is possible to apply the modified schema of chiral scalar superfield (3.2) -

$$
\begin{gathered}
E_{22}^{0}=E_{0-}^{0}=-\frac{1}{8 \square}\left(d^{2}-2 i \partial_{\rho} d^{\rho}\right) \\
\left(E_{42}^{0}\right)^{\varkappa}=\frac{1}{4 \square}\left(d-i d^{5}\right) \partial^{\varkappa}, \quad\left(E_{21}^{0}\right)_{\lambda}=-\frac{1}{4 \square}\left(d+i d^{5}\right) \partial_{\lambda} .
\end{gathered}
$$

The general equation is written in the form

$$
\square\left|\begin{array}{cc}
\left(E_{11}^{1}+E_{11}^{0}\right)_{\lambda} & a\left(E_{12}^{0}\right)^{\varkappa} \\
b\left(E_{21}^{0}\right)_{\lambda} & c E_{22}^{0}
\end{array}\right|\left|\begin{array}{c}
h^{\lambda} \\
\Phi
\end{array}\right|+m^{2}\left|\begin{array}{c}
h^{\varkappa} \\
\Phi
\end{array}\right|=0 .
$$

Taking into account the expressions of $E_{i j}^{Y}$, we get

$$
\begin{aligned}
& -\frac{1}{8}\left(d^{2}+2 i \partial_{\rho} d^{\rho}\right) h^{\kappa}+\frac{a}{4}\left(d-i d^{5}\right) \partial^{\varkappa} \Phi+m^{2} h^{\varkappa}=0, \\
& -\frac{b}{4}\left(d+i d^{5}\right) \partial_{\lambda} h^{\lambda}-\frac{c}{8}\left(d^{2}-2 i \partial_{\rho} d^{\rho}\right) \Phi+m^{2} \Phi=0 .
\end{aligned}
$$

Decomposing $\pi=\pi^{1}+\pi^{0}$ 


$$
\pi^{1}=\left|\begin{array}{cc}
E_{11}^{1} & 0 \\
0 & 0
\end{array}\right|, \quad \pi^{0}=\left|\begin{array}{cc}
E_{11}^{0} & a E_{12}^{0} \\
b E_{21}^{0} & c E_{22}^{0}
\end{array}\right|
$$

we can see that equation (5.38) describes superspin 1 with mass $m$. Superspin 0 is eliminated if $\pi^{0}$ is nilpotent, i. e. $a b$ and $c$ satisfy

$$
a b=c=-1 \text {. }
$$

If we want to describe one or two superspins 0 in addition to superspin 1, then the masses of $Y=0$ states are calculated from the nonzero eigenvalues $\lambda_{1}$ and $\lambda_{2}$ of matrix $\pi_{0}-$

$$
2 \lambda_{1,2}=c+1 \pm \sqrt{(c-1)^{2}+4 a b} .
$$

In order to have physical mass spectrum $a b$ and $c$ must satisfy

$$
c \geqslant-1, \quad a b \leqslant c, \quad(c-1)^{2}+4 a b \geqslant 0 .
$$

The analysis of mass spectrum is performed similarly as in 5.1 , and is here omitted.

The same general schema works also in the case when we choose $E_{11}^{1}$ to be $E_{1-}^{1}$. Now the operators $E_{i j}^{Y}$ are

$$
\begin{aligned}
& \left(E_{11}^{1}\right)_{\lambda}^{\kappa_{\lambda}}=-\frac{1}{8 \square}\left(d^{2}-2 i \partial_{\rho} d^{\rho}\right)\left(\eta_{\lambda}-\frac{\partial^{\varkappa} \partial_{\lambda}}{\square}\right), \\
& \left(E_{11}^{0}\right)^{\kappa_{\lambda}}=-\frac{1}{8 \square}\left(d^{2}-2 i \partial_{\rho} d^{\rho}\right) \frac{\partial^{\varkappa} \partial_{\lambda}}{\square}, \\
& E_{22}^{0}=-\frac{1}{8 \square}\left(d^{2}+2 i \partial_{\rho} d^{\rho}\right), \\
& \left(E_{12}^{0}\right)^{\kappa}=\frac{1}{4 \square}\left(d+i d^{5}\right) \partial^{\varkappa}, \quad\left(E_{21}^{0}\right)_{\lambda}=-\frac{1}{4 \square}\left(d-i d^{5}\right) \partial_{\lambda} .
\end{aligned}
$$

The corresponding equation is the following -

$$
\begin{aligned}
& -\frac{1}{8}\left(d^{2}-2 i \partial_{\rho} d^{\rho}\right) h^{\kappa}+\frac{a}{4}\left(d+i d^{5}\right) \partial^{\kappa} \Phi+m^{2} h^{\varkappa}=0, \\
& -\frac{b}{4}\left(d-i d^{5}\right) d_{\lambda} h^{\lambda}-\frac{c}{8}\left(d^{2}+2 i \partial_{\rho} d^{\rho}\right) \Phi+m^{2} \Phi=0 .
\end{aligned}
$$

The last equation concludes the investigation of possible equations, using vector superfield $h^{\mu}(x, \theta)$ and scalar superfield $\Phi(x, \theta)$. In principle it is possible to write down similar equations as in the case of chiral scalar superfield, but these equations need supplementary conditions to separate some of the superspins.

In conclusion; we would like to point out once more that the general schema for obtaining superfield equations of motion proposed in the second paragraph works well in the case of known equations, and allows to obtain new superfield equations of motion. We also hope that the formalism of superprojectors $E_{i j}^{Y}$ is useful in physical applications since the calculation of Green functions via the Klein-Gordon divisor may be performed quite easily. 


\section{A P P E N D I X}

\section{Superprojectors}

We use the following notations: $\frac{1}{2}\left\{\gamma^{\mu}, \gamma^{v}\right\}=\eta^{\mu v}=\operatorname{diag}\left(+-\frac{-}{-}\right.$, $\gamma^{5}=\gamma^{0} \gamma^{1} \gamma^{2} \gamma^{3}, \quad \sigma^{\mu v}=\frac{1}{2}\left[\gamma^{\mu}, \gamma^{v}\right], \quad \square=\partial_{\mu} \partial^{\mu} \quad$ and $\hat{\partial}=\partial_{\mu} \gamma^{\mu} . \quad$ In order to simplify the expressions which contain the covariant derivative $D_{\alpha}$ we denote

$$
d=\bar{D} D, \quad d^{5}=\bar{D} \gamma^{5} D, \quad d^{\mu}=\bar{D} i \gamma^{\mu} \gamma^{5} D .
$$

Here we list some of the mostly used expressions for $d, d^{5}$ and $\mathrm{d}^{\mu}$ [2]

$$
\begin{aligned}
& d^{2}=\left(d^{5}\right)^{2}, \quad d^{3}=-4 \square d, \quad d d^{5}=-d^{5} d=2 \partial_{\rho} d^{\rho}, \\
& d d^{\mu}=-d^{\mu} d=-2 \partial^{\mu} d^{5}, \quad d^{5} d^{\mu}=-d^{\mu} d^{5}=2 \partial^{\mu} d, \\
& d^{\mu} d^{v}=\eta^{\mu \nu} d^{2}+4\left(\square \eta^{\mu \nu}-\partial^{\mu} \partial^{v}\right)+2 \partial_{\lambda} \varepsilon^{\lambda \mu v}{ }_{\rho} d^{\rho} .
\end{aligned}
$$

We consider the superfield $\Phi_{i,}(x, \theta)$ which transforms under the Lorentz transformations according to some irreducible representation $(k, l)$ of the Lorentz group. The Poincaré spins in $(k, l)$ are $-s=$ $=k+l, k+l-1, \ldots,|k-l|$. The superspins $Y$ that correspond to each Poincaré spin $s: Y=S+1 / 2, S, S, s-1 / 2$ are extracted with the help of superprojectors

$$
\begin{aligned}
& E^{Y} \equiv E_{i i}^{Y}, \\
& E^{s+1 / 2}=\frac{1}{2 s+1}\left(1+\frac{d^{2}}{4 \square}\right)\left(s+1-\frac{1}{4 \square} \partial^{\varkappa} \varepsilon_{\varkappa \mu \rho \sigma} S^{\rho \sigma} d^{\mu}\right) P^{s}, \\
& E_{+}^{s}=-\frac{1}{8 \square}\left(d^{2}+2 i \partial_{\rho} d^{\rho}\right) P^{s}, \\
& E_{-\rightarrow}^{s}=-\frac{1}{8 \square}\left(d^{2}-2 i \partial_{\rho} d^{\rho}\right) P^{s}, \\
& E^{s-1 / 2}=\frac{1}{2 s+1}\left(1+\frac{d^{2}}{4 \square}\right)\left(s+\frac{1}{4 \square} \partial^{\kappa} \varepsilon_{\varkappa \mu \rho \sigma} S^{\rho \sigma} d^{\mu}\right) P^{s},-
\end{aligned}
$$

where $S^{\rho \sigma}$ are the Lorentz generators of representation $(k, l)$; and $P^{s}$ are projectors of Poincaré spins, + and - denote different chiral components.

Scalar superfield $\Phi(x, \theta)$

Scalar superfield reduces into three irreducible components $\Phi_{+} ; \Phi_{-}$ and $\Phi_{1 / 2}$ which are extracted with the help of projectors

$$
\begin{aligned}
& E^{1 / 2}=1+\frac{d^{2}}{4 \square} \\
& E_{+}^{0}=-\frac{1}{8 \square}\left(d^{2}+2 i \partial_{\rho} d^{\rho}\right), \\
& E_{-}^{0}=-\frac{1}{8 \square}\left(d^{2}-2 i \partial_{\rho} d^{\rho}\right) .
\end{aligned}
$$

Bispinor superfield $\psi_{\alpha}(x, \theta)$

The generators $S \rho \sigma$ are

$$
S \rho \sigma=\frac{1}{2} \sigma^{\rho \sigma} .
$$


Superspins $Y=1,1 / 2,1 / 2,0$ are extracted with the help of projectors

$$
\begin{aligned}
& E^{1}=\frac{3}{4}\left(1+\frac{d^{2}}{4 \square}\right)-\frac{1}{8 \square} \partial^{\varkappa} \sigma_{\kappa \lambda} \gamma^{5} d^{\lambda}, \\
& E_{+}^{1 / 2}=-\frac{1}{8 \square}\left(d^{2}+2 i \partial_{\rho} d^{\rho}\right), \\
& E_{-}^{1 / 2}=-\frac{1}{8 \square}\left(d^{2}-2 i \partial_{\rho} d^{\rho}\right), \\
& E^{0}=\frac{1}{4}\left(1+\frac{d^{2}}{4 \square}\right)+\frac{1}{8 \square} \partial^{\varkappa} \sigma_{\kappa \lambda} \gamma^{5} d^{\lambda} .
\end{aligned}
$$

Bispinor transforms according to the reducible representation $(1 / 2,0) \oplus(0,1 / 2)$. In order to obtain superprojectors of irreducible representations, one must add the following projectors to (A.5)

$$
P_{ \pm}^{1 / 2}=\frac{1 \pm i \gamma^{5}}{2}
$$

\section{Vector superfield $h^{\mu}(x, \theta)$}

Generators $S^{\rho \sigma}$ are

$$
\left(S^{\rho \sigma}\right)^{x_{\lambda}}=\eta^{\rho \alpha^{\sigma}} \eta^{\sigma}-\eta^{\sigma \kappa} \eta^{\rho}{ }_{\lambda},
$$

and spinprojectors $P^{1}$ and $P^{0}$

$$
\left(P^{1}\right)^{\kappa_{\lambda}}=\eta^{\kappa_{\lambda}}-\frac{\partial^{\kappa} \partial_{\lambda}}{\square}, \quad\left(P^{0}\right)^{\kappa_{\lambda}}=\frac{\partial^{\kappa} \partial_{\lambda}}{\square} .
$$

The following superprojectors correspond to the Poincaré spin 1

$$
\begin{aligned}
& \left(E_{1}^{y_{2}}\right)^{\varkappa_{\lambda}}=\frac{2}{3}\left(1+\frac{d^{2}}{4 \square}\right)\left(\eta_{\lambda}{ }-\frac{\partial^{\varkappa} \partial_{\lambda}}{\square}\right)-\frac{1}{6 \square} \varepsilon_{\lambda \rho \sigma}^{\varkappa_{\rho}} \partial^{\rho} d^{\sigma}, \\
& \left(E_{1+}^{1}\right)^{\kappa_{\lambda}}=-\frac{1}{8 \square}\left(d^{2}+2 i \partial_{\rho} d^{\rho}\right)\left(\eta^{\kappa_{\lambda}}-\frac{\partial^{\kappa} \partial_{\lambda}}{\square}\right), \\
& \left(E_{1-}^{1}\right)^{x_{\lambda}}=-\frac{1}{8 \square}\left(d^{2}-2 i \partial_{\rho} d^{\rho}\right)\left(\eta^{\kappa_{\lambda}}-\frac{\partial^{x} \partial_{\lambda}}{\square}\right), \\
& \left(E_{1}^{1 / 2}\right)_{\lambda}=\frac{1}{3}\left(1+\frac{d^{2}}{4 \square}\right)\left(\eta^{\kappa_{\lambda}}-\frac{\partial^{\varkappa} \partial_{\lambda}}{\square}\right)+\frac{1}{6 \square} \varepsilon_{\lambda, \rho \sigma}^{\alpha} \partial^{\rho} d^{\sigma},
\end{aligned}
$$

and to the Poincaré spin $0-$

$$
\begin{aligned}
& \left(E_{0}^{1 / 2}\right) x_{\lambda}=\left(1+\frac{d^{2}}{4 \square}\right) \frac{\partial^{\kappa} \partial_{\lambda}}{\square}, \\
& \left(E_{0++}^{0}\right) x_{\lambda}=-\frac{1}{8 \square}\left(d^{2}+2 i \partial_{\rho} d^{\rho}\right) \frac{\partial^{\kappa} \partial_{\lambda}}{\square} \\
& \left(E_{0-1}^{0}\right) x_{\lambda}=-\frac{1}{8 \square}\left(d^{2}-2 i \partial_{\rho} d^{\rho}\right) \frac{\partial^{\kappa} \partial_{\lambda}}{\square} .
\end{aligned}
$$




\section{REFERENCES}

1. Salam, A., Strathdee, J. Nucl. Phys., B76, № 3, 477-482 (1974).

2. Salam, A., Strathdee, J. Phys, Rev., D11, № 6, 1521-1535 (1975),

3. Wess, J. In: Current Problems in Elementary Particle and Mathematical Physics. New York, Wien, 1976.

4. Огиевецкий В. И., Сокачев Э. Мат. IV Междунар. совещания по нелокальным теориям поля, Алушта, 1976. Дубна, 1976, 183-203.

5. Ogievetsky, V. I., Sokatchev, E. J. Phys. A: Math. Gen., 10, № 11, 2021-2030 (1977).

6. Loide, R.-K., Suurvarik, P. ENSV TA Toim. Füüs. Matem., 32, № 2, 165-171 (1983).

7. Loide, R.-K., Suurvarik, P. ENSV TA Toim. Füüs. Matem., 33, № 2, 188-196 (1984).

8. Sokatchev, E. Nucl. Phys., B99, № 1, 96-108 (1975).

9. Loide, R.-K. Phys. Lett., B141, № 1, 2, 61-63 (1984).

10. Ogievetsky, V. I., Sokatchev, E. Nucl. Phys., B124, № 2, 309-316 (1977).

\section{Tallinn Technical University}

Academy of Sciences of the Estonian SSR, Institute of Physics
Received June 14,1984

\section{R.-K. LOIDE, P. SUURVARIK}

\section{SUPERVALJA LIIKUMISVORRANDID}

On antud massiga supervälja liikumisvörrandite üldine kuju superprojektsioonioperaatorite formalismis ja näidatud, et tuntud skalaarse ja bispinorsupervälja vōrrandid on pakutud üldkujul esitatavad. On toodud võimalikud võrrandid vektorsupervälja ja skalaarse supervälja jaoks. Need kirjeldavad superspinni $3 / 2$ ja madalamaid superspinne.

\section{Р.-К. ЛОИДЕ, П. СУУРВАРИК}

\section{УРАВНЕНИЯ ДВИЖЕНИЯ СУПЕРПОЛЯ}

Предложена общая форма массивных суперполевых уравнений движения в формализме суперпроекционных операторов. Показано, что известные уравнения для скалярного и биспинорного суперполей имеют предложенную структуру. Даны возможные уравнения для векторного и скалярного суперполей, огисывающие суперспин $3 / 2$ и более низкие суперспины. 